\section{Avaliação das oportunidades perdidas no
controle da transmissão vertical do HIV \\ Avaliação das oportunidades perdidas no
controle da transmissão vertical do HIV em Rio Branco, Acre, Brasil}

\author{
Evaluation of missed opportunities in the control \\ of vertical HIV transmission in Rio Branco, \\ Acre State, Brazil
}

\section{Evaluación de las oportunidades perdidas en el control de la transmisión vertical del VIH en Río Branco, Acre, Brasil}

Helena Albuquerque Catão Feitoza 1,2

Rosalina Jorge Koifman 1,3

Valeria Saraceni 4

\title{
Resumo
}

A transmissão vertical do HIV ainda representa um importante problema de saúde pública no mundo. O objetivo deste estudo foi verificar a transmissão vertical do HIV em Rio Branco, Acre, Brasil, e avaliar a possibilidade de eliminação. Foi realizado estudo transversal dos casos de HIV em gestante e longitudinal sobre a incidência da transmissão vertical do HIV na base populacional de gestantes residentes no Município de Rio Branco, no perído de 2007-2015. As coortes de gestantes foram formadas por mulheres que tiveram filhos nascidos vivos, mortos ou abortos. Os dados foram obtidos do Sistema de Informação de Agravos de Notificação (SINAN), Sistema de Informações sobre Nascidos Vivos (SINASC), Sistema de Informação sobre Mortalidade (SIM) e Sistema de Informações Hospitalares (SIH). Foi realizado o relacionamento entre as bases de dados utilizando o software OpenRecLink. Foram calculadas as prevalências de HIV em gestante, a taxa de transmissão vertical e os principais fatores associados. A prevalência de HIV em gestante apresentou tendência de aumento, e a prevalência média foi de 0,18\%, as variáveis estatisticamente associadas à ocorrência de HIV em gestantes foram idade materna $\geq 20$ anos $(p=0,007)$, menor escolaridade $(p=0,054)$ e não ter companheiro $(p=0,001)$. A transmissão vertical foi de $6,9 \%$. O uso de terapia antirretroviral (TARV) no pré-natal, mesmo entre as gestantes que já sabiam ser portadoras do vírus, foi menor que 90\%. A realização de cesáreas eletivas ficou abaixo de 60\%, e o uso de TARV no parto e pelo recém nascido nas primeiras 24 horas apresentou variações, dependendo do periodo em que o diagnóstico materno foi realizado. Embora as estratégias de eliminação da transmissão vertical do HIV estejam bem estabelecidas, os resultados deste estudo ainda apontam falhas importantes na cascata de cuidados das gestantes infectadas em Rio Branco.

HIV; Gestantes; Prevalência; Transmissão Vertical de Doença Infecciosa

\author{
Correspondência \\ H. A. C. Feitoza \\ Programa de Pós-graduação em Saúde Coletiva, Universidade \\ Federal do Acre. \\ Av. Ceará 3188, 7o BEC, Rio Branco, AC 69918-111, Brasil. \\ helenacatao2009@hotmail.com \\ 1 Programa de Pós-graduação em Saúde Coletiva, Universidade \\ Federal do Acre, Rio Branco, Brasil. \\ 2 Secretaria Municipal de Saúde de Rio Branco, Rio Branco, \\ Brasil. \\ 3 Escola Nacional de Saúde Pública Sergio Arouca, Fundação \\ Oswaldo Cruz, Rio de Janeiro, Brasil. \\ 4 Secretaria Municipal de Saúde do Rio de Janeiro, Rio de \\ Janeiro, Brasil.
}




\section{Introdução}

Apesar dos avanços científicos, ao longo dos últimos 39 anos após a descrição da aids, a transmissão vertical do HIV ainda representa um importante problema de saúde pública no mundo. Um dos elementos essenciais das orientações estratégicas da Organização Mundial da Saúde (OMS) para atingir os Objetivos de Desenvolvimento Sustentável das Nações Unidas e conter a propagação da doença é a erradicação de novas infecções por HIV em crianças 1.

No Brasil, em 2018, entre os 181 indivíduos menores de 13 anos com aids, 86,2\% tiveram como via de infecção a transmissão vertical 2. Esse resultado demonstra que, embora reconhecidos os progressos quanto a conhecimento, implantação e adesão às condutas recomendadas pelo Ministério da Saúde para a prevenção da transmissão vertical do $\mathrm{HIV}^{3}$, ainda existem lacunas importantes na rede de assistência a serem preenchidas, como a captação precoce e a adesão das gestantes ao pré-natal, bem como a adequada implantação das medidas de prevenção da transmissão vertical no pré-natal, no parto e para o recém-nascido 4,5,6,7. Dessa forma, crianças continuam sendo infectadas pelo HIV 8 .

Em 2010, os países membros da Organização Pan-Americana da Saúde (OPAS) adotaram metas para eliminar a transmissão vertical da sífilis e do HIV até o ano de 2015, visando uma redução na incidência de casos de HIV em crianças para 0,3 caso por 1.000 nascidos vivos e uma taxa de transmissão vertical de 2\%. Em 2014, a OMS reforçou esse plano, definindo os critérios para validação da eliminação da transmissão vertical da sífilis e do HIV. Para a infecção pelo HIV, os indicadores de impacto são definidos pela ocorrência de 50 novas infecções por HIV em crianças por 100 mil nascidos vivos e um taxa de transmissão $<2 \%$ nos casos sem amamentação. Como indicadores de processo, os objetivos incluem realizar, pelo menos, uma consulta pré-natal para 95\% das mulheres grávidas, pelo menos, um teste de HIV para 95\% das mulheres grávidas durante o pré-natal e uso de terapia antirretroviral (TARV) combinada por $90 \%$ das mulheres grávidas com HIV. Nas Américas, o objetivo do uso combinado da TARV por gestante infectada é de $95 \% 9,10$.

O Ministério da Saúde recomenda, como estratégias para a redução da transmissão vertical do HIV, o uso de TARV na grávida e no recém-nascido, indicação adequada do parto cesáreo e a não amamentação 3,11. Mulheres vivendo com HIV que tenham gestações planejadas e que as intervenções sejam realizadas adequadamente durante o pré-natal, o parto e a amamentação têm o risco de transmissão vertical do HIV reduzido para menos de $2 \%$. No entanto, sem o adequado planejamento e seguimento, o risco pode variar de $15 \%$ a $45 \%{ }^{3}$.

Conhecer o cenário e a situação epidemiológica do HIV na gestante e na criança torna-se de grande relevância para avaliar a possibilidade de eliminação da transmissão vertical do HIV em Rio Branco, Acre, Brasil, assim como identificar os fatores associados para sugerir estratégias para redução da transmissão vertical foi o objetivo deste estudo.

\section{Materiais e métodos}

\section{Tipo de estudo}

Estudo transversal para avaliar os fatores de riscos associados à infecção pelo HIV em gestantes e a prevalência de transmissão vertical do HIV na coorte de gestantes de Rio Branco, no período de 20072015. Também foram avaliadas as oportunidades perdidas no controle e prevenção da transmissão vertical do HIV, de acordo com o protocolo vigente no período do estudo.

\section{População do estudo}

A população do estudo foi formada pela coorte de mulheres que gestaram e concluíram seu período gestacional com filhos nascidos vivos, natimortos ou abortos, residentes no Município de Rio Branco, no período de 2007-2015, infectadas ou não pelo HIV, além dos recém-nascidos resultantes dessas gestações, que tenham sido notificados como criança exposta ao HIV ou aids em criança no Sistema de Informação de Agravos de Notificação (SINAN), durante o período do estudo. 


\section{Fonte de dados}

Os dados sobre as gestantes foram obtidos do SINASC (Sistema de Informação sobre Nascidos Vivos), SIM (Sistema de Informação sobre Mortalidade) e SIH (Sistema de Informações Hospitalares). As análises primárias dos casos de HIV em gestantes foram realizadas com base nos dados do SINAN-NET, e, posteriormente, somadas a esses os casos de HIV em gestantes identificados nos demais sistemas (SINAN-aids e SINAN-criança exposta ao HIV).

Os dados referentes ao SINASC, SIM e SINAN-NET foram disponibilizados pela Secretaria Municipal de Saúde de Rio Branco (SEMSA). Os dados do SIH foram disponibilizados pela Secretaria Estadual de Saúde do Acre (SESACRE).

As variáveis de interesse foram selecionadas dentre aquelas padronizadas nos formulários específicos para cada Sistema de Informação em Saúde: Fichas de Investigação Epidemiológica (FIE), Declaração de Nascido Vivo (DN), Declaração de Óbito (DO) e Autorização de Internação Hospitalar (AIH), sendo que, para as informações divergentes, foram priorizados os dados do SINASC, tendo em vista que a maior parte dos dados foi proveniente desse sistema, que se baseia na DN, preenchida no ato do nascimento, e serve de base para o documento cartorial Certidão de Nascimento, seguidos do SINAN e do SIM.

\section{Relacionamento entre bases de dados}

Os dados foram extraídos dos sistemas SINAN, SINASC, SIM e SIH, exportados para planilhas Microsoft Excel v. 1904 (https://products.office.com/). Utilizando o Stata v. 11.0.0 (https://www. stata.com), foi aplicado o linkage determinístico nessas bases, tendo como chave composta de agregação o "nome da mãe" + "data de nascimento". O relacionamento probabilístico das bases de dados foi realizado por meio do software OpenRecLink (http://reclink.sourceforge.net/), tendo o nome da mãe como chave primária, de maneira que, para nomes de mães semelhantes, não idênticos, foram utilizados outros métodos, além da blocagem e do pareamento, foi realizada inspeção visual (nome da gestante, nome da mãe, idade da gestante, idade da mãe, ano da notificação, ano do óbito, bairro, dependendo da disponibilidade nas bases originais). Finalmente, os dados foram analisados por meio do software Statistical Package for the Social Sciences (SPSS - https://www.ibm.com/), em sua 17a versão para o Windows.

\section{Análise estatística}

Foi realizada a análise descritiva, com o uso de medidas de tendência central e dispersão para as variáveis contínuas e uso de medidas de frequência para as variáveis categóricas. Posteriormente, foi utilizado o teste de normalidade Kolmogorov-Smirnov para as variáveis contínuas, a fim de definir o método de comparação a ser utilizado nessas variáveis, sendo a comparação entre os grupos feita pelo teste $\mathrm{t}$ de Student, quando a variável apresentou uma distribuição aproximadamente normal, e pelo teste não paramétrico de Mann-Whitney, quando a variável apresentou comportamento não normal. Para as variáveis categóricas, o teste de comparação utilizado foi o qui-quadrado de Pearson. Para todos eles, adotou-se um erro alfa admissível de 0,05.

Para estimar a prevalência de HIV em gestantes, foram calculadas as medidas de frequência do tipo prevalência, utilizando-se o número de gestantes HIV positivas sobre o número de gestantes com filhos nascidos vivos. Para as análises bivariadas, foram estimadas as medidas de associação do tipo razão de chances (odds ratio) com intervalos de 95\% de confiança (IC95\%), seguidas de uma análise multivariada, com uso da regressão logística, a fim de explorar a influência dos fatores associados, levando em consideração, para entrada no modelo, as variáveis sociodemográficas relacionadas às gestantes que apresentaram associação significativa na análise bivariada.

A transmissão vertical do HIV foi calculada utilizando, no numerador, a quantidade de casos de aids em crianças menores de cinco anos decorrentes de transmissão vertical e, no denominador, os casos de gestantes HIV positivas. 


\section{Aspectos éticos}

O projeto foi aprovado pelo Comitê de Ética em Pesquisa do Hospital das Clínicas do Acre (HCA/ FUNDHACRE, parecer no 1.899.737/2017). A privacidade e a confidencialidade das participantes do estudo foram asseguradas em todas as fases da pesquisa.

\section{Resultados}

No período de 2007-2015, foram notificados, no SINAN-HIV em gestante, 84 casos residentes em Rio Branco. Nesse mesmo período, foram identificados seis casos de aids em crianças, em que três mães não tinham notificação no banco de HIV em gestante. No SINAN-aids, foram identificadas 16 mulheres com a informação de gravidez no momento da notificação, sendo que seis não tinham notificação no banco de HIV em gestante. Dentre os 79 casos de crianças expostas ao HIV notificados no SINAN, foi verificado que 21 mães não tinham notificação no SINAN-HIV em gestante. Após o relacionamento entre os bancos de dados do SINAN, formou-se uma coorte de 114 gestantes infectadas pelo HIV (Figura 1).

Das 114 gestantes HIV positivas identificadas nas bases de dados do SINAN-HIV/aids, após linkage com o SINASC, SIM e SIH (2007 a 2016), nove não eram residentes de Rio Branco, e 14 não foram localizadas. Das 91 gestantes identificadas, 90 estavam no SINASC, e uma, no SIM. No SINASC, das 90 gestantes, 13 vieram do SINAN-HIV em gestante, 15 do SINAN-criança exposta ao HIV, duas do SINAN-aids em criança e duas do SINAN-aids em mulheres. Também foram identificadas 46 gestantes que constavam simultaneamente nos bancos SINAN-HIV em gestante e criança exposta ao HIV; três no SINAN-criança exposta ao HIV e aids em mulheres; duas no SINAN-criança exposta ao HIV e aids em criança; seis no SINAN-HIV em gestante, criança exposta ao HIV e aids em mulheres; e uma no SINAN-HIV em gestante, criança exposta ao HIV e aids em criança (Figura 1).

Em Rio Branco, no período de 2007-2015, de um total de 61.626 gestantes que tiveram filhos nascidos vivos, $84(0,14 \%)$ estavam infectadas pelo HIV. Entretanto, após o linkage dos diversos bancos de dados, foi identificada uma prevalência média estimada de $0,18 \%$, o que representa um aumento de $22,2 \%$ em relação à prevalência média observada. Tanto a prevalência observada quanto a estimada apresentaram tendência de aumento, saindo de 0,04\% e 0,07\% em 2007 para 0,30\% e 0,46\% em 2015, um incremento de $86,6 \%$ e $84,8 \%$ respectivamente (Figura 2).

Para a análise das coortes de gestantes (SINASC, SIM e SIH), foi incluído o ano de 2016, totalizando 67.891 nascidos vivos, por conter informações referentes às variáveis de interesse das gestantes notificadas no SINAN para HIV no fim de 2015.

$\mathrm{Na}$ Tabela 1 , quanto às variáveis obstétricas, na análise univariada, as gestantes HIV positivas tiveram menor assistência pré-natal $(\mathrm{p}=0,031)$ e, como consequência da infecção pelo HIV, apresentaram maior proporção de parto cesáreo $(\mathrm{p}<0,001)$. A prematuridade $(\mathrm{p}=0,431)$ e o baixo peso $(\mathrm{p}=0,049)$ foram mais prevalentes entre as gestantes HIV positivas, porém sem significância estatística. Por meio da análise multivariada, as variáveis sociodemográficas estatisticamente associadas à ocorrência de HIV em gestantes na coorte de mães de nascidos vivos foram: a idade materna $\geq 20$ anos ( $p=0,007)$, menor escolaridade $(\mathrm{p}=0,054)$ e não ter companheiro $(\mathrm{p}=0,001)$.

De acordo com as notificações de gestantes HIV positivas em Rio Branco (84 casos), 53 (63,1\%) tinham entre 20 e 29 anos, 54 (64,3\%) notificações ocorreram no 3o trimestre de gestação, 52 (61,9\%) tinham mais de 8 anos de escolaridade, 67 (79,8\%) eram pardas, 34 (40,5\%) já tinham o diagnóstico antes do pré-natal, $72(85,7 \%)$ realizaram pré-natal, e 63 (75\%) tiveram seus filhos nascidos vivos (Tabela 2).

No período do estudo, foram notificados seis casos de aids em crianças menores de cinco anos, em decorrência de transmissão vertical, entre as quais, somente três tinham mães notificadas como gestante HIV positiva. Para calcular a transmissão vertical, foram consideradas, no numerador, as seis crianças notificadas no banco aids em criança e, no denominador, as 84 gestantes já notificadas no banco de HIV em gestante mais as três mães do banco de aids em criança não notificadas no SINAN-HIV em gestante, totalizando 87 gestantes ( $84+3$ mães não notificadas), o que representou uma transmissão vertical de 6,9\%. Entretanto, levando em consideração que, após o linkage, foram 


\section{Figura 1}

Fluxograma dos casos de gestantes HIV positivas identificados no Sistema de Informação de Agravos de Notificação (SINAN), Sistema de Informação sobre Nascidos Vivos (SINASC), Sistema de Informação sobre Mortalidade (SIM) e Sistema de Informações Hospitalares (SIH). Rio Branco, Acre, Brasil, 2007-2015.

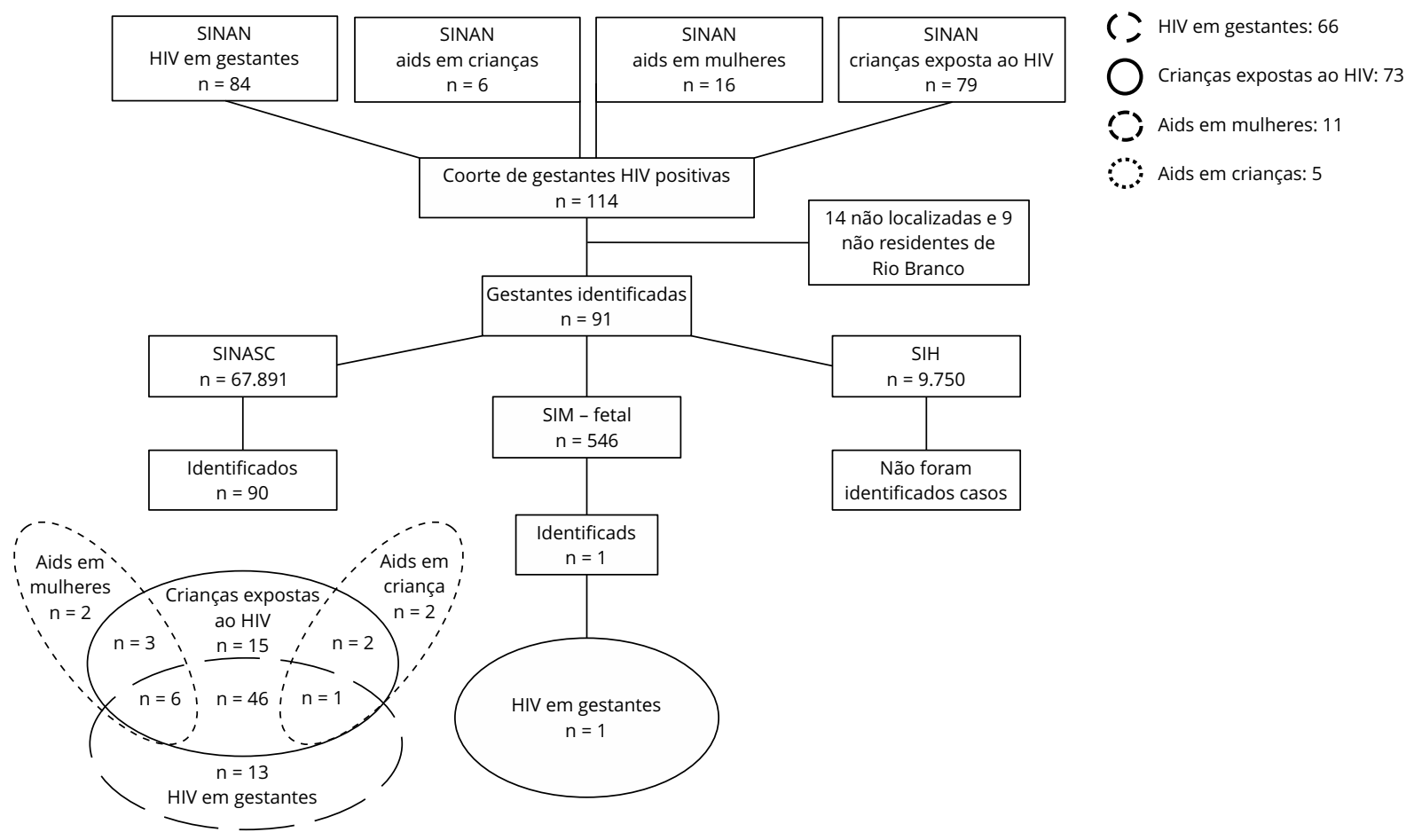

Fonte: SIM; SINAN; SINASC; SIH; Secretaria Municipal de Saúde de Rio Branco.

identificadas, nos vários bancos analisados, 91 gestantes HIV positivas residentes de Rio Branco e localizadas no SINASC ou SIM, a transmissão vertical foi reduzida para 6,6\%.

Na Tabela 3, encontram-se as oportunidades de redução da transmissão vertical do HIV em Rio Branco a partir do momento do diagnóstico do HIV (antes da gestação, durante o pré-natal ou na admissão para o parto) das gestantes e de acordo com a realização do pré-natal. Das gestantes que fizeram pré-natal e já tinham o diagnóstico prévio do HIV, 85,7\% fizeram uso da TARV durante o pré-natal e o parto, 53,6\% tiveram como via de parto a cesárea eletiva, e 85,7\% dos recém-nascidos receberam TARV nas primeiras 24 horas de vida. Das gestantes que tiveram o diagnóstico do HIV no pré-natal, 63,3\% fizeram uso da TARV durante o pré-natal, 56,7\% fizeram uso de TARV no parto, 50\% tiveram como via de parto a cesárea eletiva, e 56,7\% dos recém-nascidos receberam TARV nas primeiras 24 horas de vida. No caso das gestantes com diagnóstico somente na hora do parto, verificou-se que $92,9 \%$ fizeram uso de TARV no parto, 28,6\% tiveram como via de parto a cesárea eletiva, e 100\% dos recém-nascidos receberam TARV nas primeiras 24 horas de vida. 
Figura 2

Prevalências observadas e estimadas de gestantes HIV positivas. Rio Branco, Acre, Brasil, 2007 a 2015.

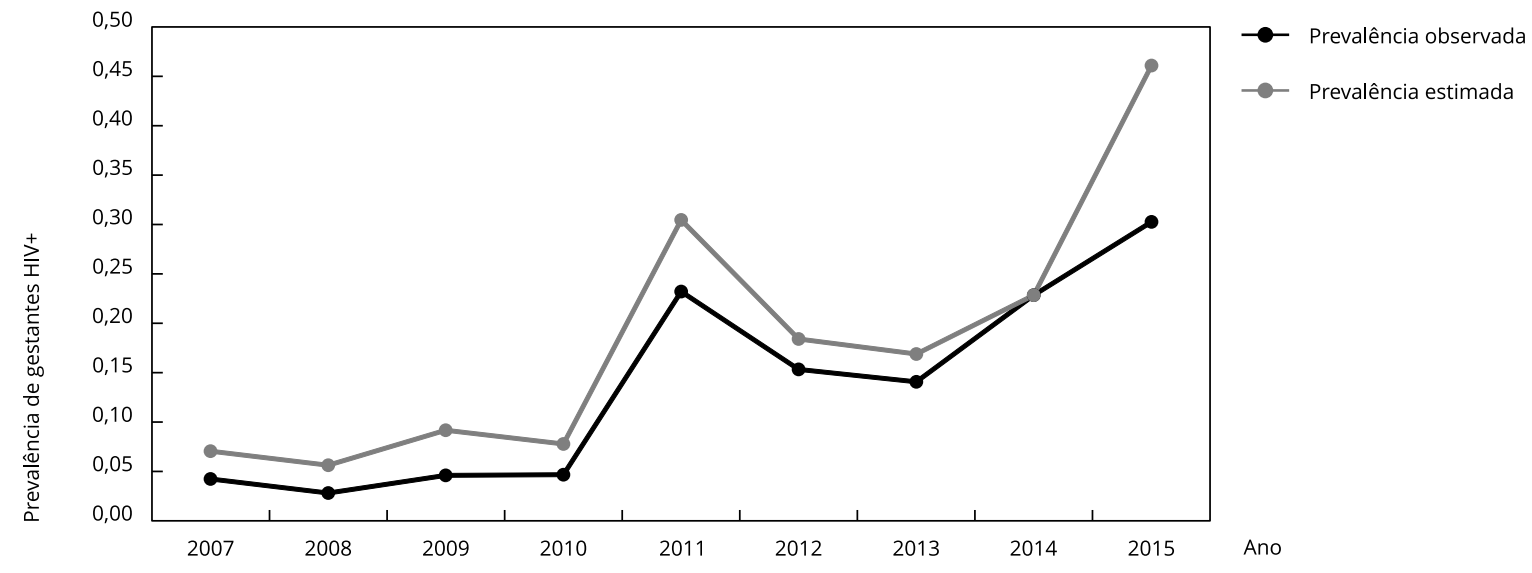

Fonte: Sistema de Informação de Agravos de Notificação; Secretaria Municipal de Saúde de Rio Branco.

\section{Discussão}

Em Rio Branco, a prevalência média observada e estimada de HIV em gestantes, entre 2007-2015, foi de $0,14 \%$ e $0,18 \%$ respectivamente, tais prevalências foram inferiores à identificada no Brasil para o ano de 2017 (0,28\% 12. Entretanto, assim como no país, Rio Branco apresentou tendência de aumento, saindo de 0,07\% em 2007 para 0,46\% em 2015. Esse aumento poderia ser explicado, em parte, pela ampliação do diagnóstico no pré-natal com a introdução dos testes rápidos de rotina 12 . Entre os anos de 2008 a 2015, Rio Branco apresentou um aumento de 2.293,1\% na realização de testes rápidos em gestantes 13. Prevalências superiores foram verificadas em diversos estudos 14,15,16,17,18,19,20,21.

No Município de Rio Branco, as gestantes infectadas pelo HIV eram mais velhas, semelhante ao encontrado em alguns estudos nacionais 21,22 . Assim como em outros estudos, apresentaram menor escolaridade 22,23 e não tinham companheiro, como identificado em 30,7\% das gestantes do estudo Nascer no Brasil 22 e em um estudo realizado em Camarões, onde mulheres casadas ou vivendo com o parceiro tiveram menos probabilidade de ser infectadas que mulheres solteiras 24 .

No SINAN-gestante HIV positiva, $85,7 \%$ das gestantes realizaram pré-natal, essa cobertura de pré-natal foi semelhante à encontrada em seis estados brasileiros entre os anos de 2007-2012, com variação de cobertura entre 79 e $91 \%$ 23. Entretanto, abaixo da cobertura encontrada em um estudo nacional de $95,8 \% 22$.

Vale ressaltar que $23,8 \%$ das mulheres que fizeram parte deste estudo tiveram o diagnóstico do HIV no momento do parto, semelhante ao encontrado no Amazonas, onde quase 20\% das mulheres chegaram ao parto sem saber seu status HIV 23 e aos $29 \%$ das mulheres que não foram testadas durante o pré-natal em outro estudo nacional 25. A não detecção do HIV no pré-natal é uma oportunidade perdida de intervenção na grávida portadora do vírus, aumentando as possibilidades da ocorrência de transmissão vertical.

Ao comparar os resultados da transmissão vertical de 6,9\% em Rio Branco, foram verificados resultados inferiores em Belo Horizonte, Minas Gerais (3\%) 26 e no Rio Grande do Sul (4,9\%) 27. Bem como valores superiores foram identificados em Pernambuco (9,2\%) 28 e no Amazonas (9,9\%) 29.

As falhas na cascata de cuidado para a prevenção da transmissão vertical do HIV em Rio Branco se fizeram presentes, independente do cenário no qual o diagnóstico foi realizado. Dentre as gestantes que realizaram pré-natal, foi identificado que o uso de TARV na gestação foi inferior a 90\%, mesmo entre as gestantes que já tinham conhecimento de serem portadoras do HIV antes da gestação atual, 
Tabela 1

Características sociodemográficas e obstétricas das gestantes HIV positivas na coorte de nascidos vivos. Rio Branco, Acre, Brasil, 2007-2015.

\begin{tabular}{|c|c|c|c|c|c|c|}
\hline \multirow[t]{2}{*}{ Características } & \multicolumn{6}{|c|}{ HIV em gestante } \\
\hline & $\begin{array}{c}\operatorname{Sim} \\
{[n=90(\%)]}\end{array}$ & $\begin{array}{c}\text { Não } \\
{[n=67.801(\%)]}\end{array}$ & OR (IC95\%) * & Valor de $p$ & OR ajustada (IC95\%) & Valor de $p$ \\
\hline \multicolumn{7}{|l|}{ Idade materna (anos) } \\
\hline$\geq 20$ & $79(87,8)$ & $51.790(76,4)$ & $2,2(1,18-4,17)$ & 0,011 & $2,4(1,27-4,53)$ & 0,07 \\
\hline$<20$ & $11(12,2)$ & $16.011(23,6)$ & 1,00 & & 1,00 & \\
\hline \multicolumn{7}{|l|}{ Escolaridade (anos) } \\
\hline$>8$ & $78(86,7)$ & $62.137(92,3)$ & 1,00 & 0,029 & 1,00 & 0,054 \\
\hline$\leq 8$ & $12(13,3)$ & $4.918(7,3)$ & $1,9(1,06-3,57)$ & & $1,8(0,99-3,36)$ & \\
\hline Ignorado & $0(0,0)$ & $746(0,4)$ & - & & - & \\
\hline \multicolumn{7}{|l|}{ Cor da pele da mãe ** } \\
\hline Branca & $7(9,1)$ & $3.695(9,1)$ & 1,00 & & - & - \\
\hline Preta & $3(3,9)$ & $528(1,3)$ & $3,0(0,77-11,63)$ & 0,112 & & \\
\hline Amarela & $0(0,0)$ & $171(0,4)$ & - & 0,996 & & \\
\hline Parda & $56(72,7)$ & $28.503(70,1)$ & $1,0(0,47-2,28)$ & 0,928 & & \\
\hline Indígena & $0(0,0)$ & $106(0,3)$ & - & 0,997 & & \\
\hline Ignorado & $11(14,3)$ & $7.651(18,8)$ & - & & & \\
\hline \multicolumn{7}{|l|}{ Estado civil } \\
\hline Sem companheiro & $29(32,2)$ & $13.619(20,1)$ & $1,9(1,21-2,95)$ & 0,004 & $2,1(1,33-3,26)$ & 0,001 \\
\hline Com companheiro & $60(66,7)$ & $53.351(78,7)$ & 1,00 & & 1,00 & \\
\hline Ignorado & $1(1,1)$ & $831(1,2)$ & - & & & \\
\hline \multicolumn{7}{|l|}{ Assistência pré-natal } \\
\hline Sim & $81(90,0)$ & $65.239(96,2)$ & 1,00 & 0,03 & - & - \\
\hline Não & $6(6,7)$ & $1.813(2,7)$ & $2,7(1,16-6,12)$ & & & \\
\hline Ignorado & $3(3,3)$ & $749(1,1)$ & - & & & \\
\hline \multicolumn{7}{|c|}{ Número de consultas de pré-natal } \\
\hline$\geq 7$ & $41(45,6)$ & $30.294(44,7)$ & 1,00 & 0,72 & - & - \\
\hline$<7$ & $46(51,1)$ & $36.758(54,2)$ & $1,1(0,71-1,65)$ & & & \\
\hline Ignorado & $3(3,3)$ & $749(1,1)$ & - & & & \\
\hline \multicolumn{7}{|c|}{ Trimestre que iniciou o pré-natal ** } \\
\hline 10 & $28(36,4)$ & $18.643(45,9)$ & 1,00 & & - & - \\
\hline 20 & $13(16,9)$ & $8.058(19,8)$ & $1,1(0,56-2,07)$ & 0,831 & & \\
\hline 3o & $6(7,8)$ & $2.300(5,6)$ & $1,7(0,72-4,20)$ & 0,220 & & \\
\hline Ignorado & $30(38,9)$ & $11.653(28,7)$ & - & & & \\
\hline \multicolumn{7}{|l|}{ Tipo de parto } \\
\hline Vaginal & $20(22,2)$ & $37.629(55,5)$ & 1,00 & $<0,001$ & - & - \\
\hline Cesáreo & $70(77,8)$ & $30.121(44,4)$ & $4,4(2,66-7,17)$ & & & \\
\hline Ignorado & $0(0,0)$ & $51(0,1)$ & - & & & \\
\hline \multicolumn{7}{|l|}{ Prematuridade } \\
\hline Sim & $10(11,1)$ & $6.137(9,1)$ & $1,3(0,67-2,53)$ & 0,431 & - & - \\
\hline Não & $72(80,0)$ & $57.595(84,9)$ & 1,00 & & & \\
\hline Ignorado & $8(8,9)$ & $4.069(6,0)$ & - & & & \\
\hline \multicolumn{7}{|l|}{ Baixo peso ao nascer } \\
\hline Sim & $13(14,4)$ & $5.832(8,6)$ & $1,8(0,99-3,23)$ & 0,049 & - & - \\
\hline Não & $77(85,6)$ & $61.862(91,2)$ & 1,00 & & & \\
\hline Ignorado & $0(0,0)$ & $107(0,2)$ & - & & & \\
\hline
\end{tabular}

IC95\%: intervalo de 95\% de confiança; OR: odds ratio.

Fonte: Sistema de Informação de Agravos de Notificação; Sistema de Informação sobre Nascidos Vivos; Secretaria Municipal de Saúde de Rio Branco.

* Teste qui-quadrado $\left(\chi^{2}\right)$ de Pearson;

** Variáveis disponíveis na Declaração de Nascido Vivo a partir de 2011. 
Tabela 2

Características dos casos de gestantes HIV positivas notificados no Sistema de Informação de Agravos de Notificação. Rio Branco, Acre, Brasil, 2007-2015.

\begin{tabular}{|c|c|c|}
\hline Variáveis & $n(N=84)$ & $\%$ \\
\hline \multicolumn{3}{|l|}{ Idade (anos) } \\
\hline $10-19$ & 12 & 14,3 \\
\hline $20-29$ & 53 & 63,1 \\
\hline $30-39$ & 18 & 21,4 \\
\hline $40-49$ & 1 & 1,2 \\
\hline \multicolumn{3}{|c|}{ Trimestre gestacional da notificação } \\
\hline $1 \underline{0}$ & 11 & 13,1 \\
\hline 20 & 18 & 21,4 \\
\hline 3o & 54 & 64,3 \\
\hline Ignorado & 1 & 1,2 \\
\hline \multicolumn{3}{|l|}{ Escolaridade (anos) } \\
\hline$>8$ & 52 & 61,9 \\
\hline$\leq 8$ & 30 & 35,7 \\
\hline Ignorado & 2 & 2,4 \\
\hline \multicolumn{3}{|l|}{ Cor da pele } \\
\hline Branca & 11 & 13,1 \\
\hline Preta & 4 & 4,8 \\
\hline Amarela & 1 & 1,2 \\
\hline Parda & 67 & 79,8 \\
\hline Indígena & 1 & 1,2 \\
\hline \multicolumn{3}{|l|}{ Diagnóstico do HIV } \\
\hline Antes do pré-natal & 34 & 40,5 \\
\hline Durante o pré-natal & 30 & 35,7 \\
\hline Durante o parto & 20 & 23,8 \\
\hline \multicolumn{3}{|l|}{ Realizou pré-natal } \\
\hline Sim & 72 & 85,7 \\
\hline Não & 11 & 13,1 \\
\hline Ignorado & 1 & 1,2 \\
\hline \multicolumn{3}{|l|}{ Evolução da gravidez } \\
\hline Vivo & 63 & 75,0 \\
\hline Natimorto & 1 & 1,2 \\
\hline Aborto & 5 & 6,0 \\
\hline Não se aplica & 3 & 3,5 \\
\hline Ignorado & 12 & 14,3 \\
\hline
\end{tabular}

Fonte: Sistema de Informação de Agravos de Notificação; Secretaria Municipal de Saúde de Rio Branco.

provavelmente pelo início tardio do pré-natal, já que as notificações das gestantes HIV positivas no SINAN ocorrem em sua maioria no 3o da gestação (64,3\%). A TARV, quando iniciada a partir da 14a de gestação, pode resultar na infecção do concepto, devido à exposição prolongada à alta carga viral 30. Estudo realizado em Santa Catarina verificou que a não utilização de TARV durante a gestação e o aleitamento materno foram os fatores estatisticamente associados à soroconversão para a infecção pelo HIV no recém-nascido 31. Esses resultados refletem falhas nos processos de captação precoce e seguimento das gestantes, apontando lacunas a serem trabalhadas em relação aos cuidados preconizados pelo Ministério da Saúde.

A proporção do uso de TARV no parto e pelo recém-nascido nas primeiras 24 horas também ficou abaixo do ideal em todos os cenários, com exceção do uso de TARV no parto e pelo recém-nascido nas primeiras 24 horas, quando o diagnóstico materno ocorreu no momento do parto, tendo em vista 
Tabela 3

Distribuição proporcional da cascata de cuidados para a profilaxia da transmissão vertical do HIV, de acordo com o momento do diagnóstico do HIV materno e assistência pré-natal. Rio Branco, Acre, Brasil, 2007-2015.

\begin{tabular}{|c|c|c|}
\hline \multirow[t]{2}{*}{ Cascata de cuidados } & \multicolumn{2}{|c|}{ Assistência pré-natal } \\
\hline & $\operatorname{Sim}(\%)$ & Não (\%) \\
\hline Diagnóstico do HIV antes do pré-natal & $n=28$ & $\mathrm{n}=6$ \\
\hline TARV durante o pré-natal & $24(85,7)$ & $0(0,0)$ \\
\hline TARV durante o parto & $24(85,7)$ & $1(16,7)$ \\
\hline Cesárea eletiva & $15(53,6)$ & $0(0,0)$ \\
\hline TARV no recém-nascido nas primeiras 24 horas de vida & $24(85,7)$ & $3(50,0)$ \\
\hline Diagnóstico do HIV no pré-natal & $n=30$ & $\mathrm{n}=0$ \\
\hline TARV durante o pré-natal & $19(63,3)$ & - \\
\hline TARV durante o parto & $17(56,7)$ & - \\
\hline Cesárea eletiva & $15(50,0)$ & - \\
\hline TARV no recém-nascido nas primeiras 24 hoas de vida & $17(56,7)$ & - \\
\hline Diagnóstico do HIV durante o parto & $n=14$ & $\mathrm{n}=6$ \\
\hline TARV durante o parto & $13(92,9)$ & $3(50,0)$ \\
\hline Cesárea eletiva & $4(28,6)$ & $2(33,3)$ \\
\hline TARV no recém-nascido nas primeiras 24 horas de vida & $14(100,0)$ & $4(66,7)$ \\
\hline
\end{tabular}

TARV: terapia antirretroviral.

Fonte: Sistema de Informação de Agravos de Notificação; Secretaria Municipal de Saúde de Rio Branco.

que a ficha de atendimento na maternidade pode ter mais informações sobre o parto e o nascimento do que sobre o pré-natal, provavelmente pela ausência dessas informações na caderneta de pré-natal da gestante, fato que chama a atenção para a possiblidade de falhas na integração entre os serviços de assistência pré-natal e a atenção ao parto; além disso, a maior parte das fichas de notificação foi preenchida nas maternidades (75\%), ocorrendo um viés de preenchimento das informações de condutas de protocolo para o momento do parto e do nascimento, em detrimento da informação do que ocorreu no pré-natal e no atendimento ao HIV/aids dessas gestantes.

Resultados semelhantes foram encontrados nas seis unidades da federação brasileira, onde houve destaque negativo aos cuidados realizados nas regiões Norte e Nordeste 23 . Em um estudo realizado na região metropolitana do Rio de Janeiro, das mulheres atendidas em um hospital de referência a gestantes HIV positivas, apenas 52,7\% receberam todos os cuidados necessários para a prevenção da transmissão vertical do HIV 32. Na China entre 2003 e 2011, foi verificada uma queda da transmissão vertical de $12,9 \%$ para 2,3\% dentre as gestantes que receberam adequadamente a cascata de cuidados, porém, ao incluir as mulheres que não foram adequadamente acompanhadas, esses valores subiram, respectivamente, para $27,1 \%$ e $11,5 \% 33$.

Embora o protocolo clínico oriente que as gestantes que apresentem carga viral indetectável próxima ao parto não sejam submetidas à cesárea eletiva, e sim que o parto transcorra pela indicação obstétrica 3, encontramos alto percentual de cesárea neste estudo. Como trabalhou-se com os dados do SINAN, que não informam a carga viral próxima ao parto, não foi possível avaliar se as cesáreas foram indicadas corretamente. Outro indicativo da incerteza, que ronda a indicação de cesárea nessa população, foi a menor proporção de realização de cesárea eletiva nas mulheres com infecção pelo HIV detectada no momento do parto. Além disso, não foi possível saber a situação de evolução do trabalho de parto dessas últimas, no momento do diagnóstico, o que não permitiu uma avaliação concreta do tipo de parto indicado em Rio Branco para as gestantes HIV positivas.

Convém enfatizar que os resultados deste estudo mostram um pior desempenho dos protocolos para prevenção da transmissão vertical, no momento do parto, para as mulheres sem assistência pré- 
natal, levando em consideração que essas mulheres tiveram parto hospitalar, tornando-se, portanto, imprescindível questionar a qualidade da assistência durante e após o parto, nesses estabelecimentos de saúde, a essas gestantes que provavelmente representam um grupo mais vulnerável, com acesso mais limitado aos serviços de saúde durante a gestação. Porém, mesmo que as medidas de prevenção no parto sejam tardias, ainda existem possibilidades importantes de prevenção da transmissão vertical, já que a maior parte da transmissão vertical do HIV ocorre no periparto ${ }^{3}$, daí a importância do precoce e adequado acompanhamento das gestantes HIV positivas e dos seus filhos.

De acordo com o Ministério da Saúde, quando todas as intervenções profiláticas são realizadas adequadamente, a taxa de recém-nascidos infectados é reduzida a menos de $2 \%{ }^{3}$. Nessa perspectiva, estudos têm evidenciado que é possível eliminar a transmissão vertical do HIV. Cuba, em 2015, foi o primeiro país a receber a validação da OMS quanto à eliminação da transmissão vertical do HIV 34. Na Tailândia, a prevalência do HIV na gestação foi reduzida de 2\%, na década de 1990, para 0,6\%, em 2015, e a transmissão vertical reduziu de $20 \%$ a níveis menores que $2 \%$, devido ao uso efetivo de TAVR combinada, independente do CD4 materno, além da alta cobertura dos serviços de prénatal 35. O Estado de São Paulo vem apresentando, ao longo dos anos, grande sucesso em suas ações programáticas quanto ao controle da transmissão vertical do HIV, estudos revelam que, em 1998, o estado apresentava uma taxa de 16\% 36, reduzindo, em 2006, para 2,7\% 37, no ano de 2019, o Município de São Paulo alcançou a meta de eliminação da transmissão vertical do HIV 38. Diversos países vêm trabalhando para o alcance da meta de eliminação da transmissão vertical do HIV, entretanto, as características regionais, sociais e culturais, bem como a capacidade instalada do sistema de saúde local podem afetar o alcance desse objetivo.

O seguimento das crianças expostas ao HIV é considerado uma ação de extrema importância no controle da transmissão vertical, mas que ainda constitui, na prática, uma grande barreira no sucesso das ações programáticas, até mesmo pelo fato de as fichas de investigação de criança exposta ao HIV não serem registradas no SINAN, o que acaba gerando indiretamente uma não obrigação quanto ao acompanhamento adequado dessas crianças pelo sistema de saúde. Estudos têm evidenciado que existem lacunas no acompanhamento das crianças expostas ao HIV quanto a acesso e adesão aos serviços especializados, além da falta de orientação dos familiares quanto ao processo dos cuidados necessários 23,39,40.

As limitações deste estudo estão relacionadas principalmente ao uso de dados secundários evidenciados pela ausência de informações em campos de grande importância, o que, de certa forma, pode ter influenciado em sua análise. Ao desenhar o projeto, não se pensou na inclusão do Sistema de Controle Logístico de Medicamentos (SICLOM), sistema esse que poderia ter sido uma fonte de informação a mais para encontrar as gestantes portadoras do HIV, fato que pode ter limitado nossos achados. Os resultados podem ter sido subestimados, porém são essenciais para demonstrar a complexidade dessa temática. Entretanto, o uso do linkage procurou minimizar essa situação, uma vez que vem se tornando um processo essencial na busca pela completitude dos bancos de dados dos sistemas de informação em saúde, permitindo uma melhor visualização do panorama da situação de saúde de uma população.

\section{Conclusão}

Embora as estratégias de eliminação da transmissão vertical do HIV estejam bem estabelecidas, os resultados deste estudo ainda apontam falhas importantes na cascata de cuidados das gestantes infectadas em Rio Branco. Para mudar esse cenário, faz-se necessário fortalecer, no município, a rede de assistência à saúde materno-infantil, por meio das linhas de cuidado e de prevenção das IST/aids, com aumento da testagem das gestantes na atenção primária e o encaminhamento, em tempo hábil, para a referência de tratamento das gestantes HIV positivas. A articulação com as duas maternidades que atendem ao Sistema Único de Saúde (SUS) também é prioritária para a correta atenção ao parto e ao nascimento, acoplada ao fornecimento do leite substituto, à profilaxia com TARV do recém-nascido e ao seguimento das crianças expostas como rotina são pilares essenciais para o alcance da eliminação da transmissão vertical. 


\section{Colaboradores}

H. A. C. Feitoza contribuiu para concepção do projeto de pesquisa, aquisição, análise e interpretação de dados, redação do trabalho, revisão crítica do conteúdo intelectual relevante e aprovação final da versão a ser publicada. R. J. Koifman contribuiu para concepção do projeto de pesquisa, interpretação de dados, redação do trabalho, revisão crítica do conteúdo intelectual relevante e aprovação final da versão a ser publicada. V. Saraceni contribuiu com a análise e interpretação de dados, redação do trabalho, revisão crítica do conteúdo intelectual relevante e aprovação final da versão a ser publicada.

\section{Informações adicionais}

ORCID: Helena Albuquerque Catão Feitoza (00000003-0133-2640); Rosalina Jorge Koifman (00000002-2746-7597); Valeria Saraceni (0000-00017360-6490).

\section{Agradecimentos}

À Universidade Federal do Acre; Secretaria Estadual de Saúde do Acre; Secretaria Municipal de Saúde de Rio Branco, Acre, Departamento de Vigilância Epidemiológica e Ambiental, Divisão de Informação e Análise de Dados e Coordenação de IST/aids de Rio Branco, Acre, nas pessoas da Sra. Socorro Martins e do Sr. Kennedy Albuquerque.

\section{Referências}

1. World Health Organization; Joint United Nations Programme on HIV/AIDS. Global Report: UNAIDS report on the global AIDS epidemic 2019. Geneva: World Health Organization; 2019.

2. Departamento de Doenças de Condições Crônicas e Infecções Sexualmente Transmissíveis, Secretaria de Vigilância em Saúde, Ministério da Saúde. HIV/aids 2019. Boletim Epidemiológico Especial 2019; N. especial.

3. Departamento de Doenças de Condições Crônicas e Infecções Sexualmente Transmissíveis, Secretaria de Vigilância em Saúde, Ministério da Saúde. Protocolo Clínico e Diretrizes Terapêuticas para Prevenção da Transmissão Vertical de HIV, Sífilis e Hepatites Virais. Brasília: Ministério da Saúde; 2019.

4. Cavalcante MS, Silveira ACB, Ribeiro AMS, Ramos Júnior AN. Prevention of vertical transmission of HIV: analysis of the adhesion to prophylactic measures in a reference maternity in the city of Fortaleza, State of Ceará, Brasil. Rev Bras Saúde Mater Infant 2008; 8:473-9.

5. Ramos Júnior AN, Matida LH, Saraceni V, Veras MAS, Pontes RJS. Control of mother-tochild transmission of infectious diseases in Brazil: progress in HIV/AIDS and failure in congenital syphilis. Cad Saúde Pública 2007; 23 Suppl 3:S370-8.

6. Fernandes RCSC, Araújo LC, Medina-Acosta E. O desafio da prevenção da transmissão vertical do HIV no Município de Campos dos Goytacazes, Rio de Janeiro, Brasil. Cad Saúde Pública 2005; 21:1153-9.

7. Cechim PL, Perdomini FRI, Quaresma LM. Gestantes HIV positivas e sua não adesão à profilaxia no pré-natal. Rev Bras Enferm 2007; 60:519-23.

8. Darmont MQR, Martins HS, Calvet GA, Deslandes SF, Menezes JA. Adesão ao pré-natal de mulheres HIV+ que não fizeram profilaxia da transmissão vertical: um estudo sociocomportamental e de acesso ao sistema de saúde. Cad Saúde Pública 2010; 26:1788-96.

9. Pan American Health Organization. Elimination of mother-to-child transmission of HIV and syphilis in the Americas. Update 2016. Washington DC: Pan American Health Organization; 2017.

10. World Health Organization. Global guidance on criteria and processes for validation: elimination of mother-to-child transmission (EMTCT) of HIV and syphilis. Geneva: World Health Organization; 2014.

11. Departamento de Doenças de Condições Crônicas e Infecções Sexualmente Transmissíveis, Secretaria de Vigilância em Saúde, Ministério da Saúde. Recomendações para profilaxia da transmissão vertical do HIV e terapia antirretroviral em gestantes. Brasília: Ministério da Saúde; 2010. 
12. Departamento de Doenças de Condições Crônicas e Infecções Sexualmente Transmissíveis, Secretaria de Vigilância em Saúde, Ministério da Saúde. HIV/aids - 2018. Boletim epidemiológico 2018; 49(53).

13. Departamento de Doenças de Condições Crônicas e Infecções Sexualmente Transmissíveis, Secretaria de Vigilância em Saúde, Ministério da Saúde. Indicadores e dados básicos de gestantes nos municípios brasileiros com mais de 100.000 habitantes. http://indicadoresgestantes.aids.gov.br (acessado em 14/Fev/2020).

14. Vieira ACBC, Miranda AE, Vargas PRM, Maciel ELN. Prevalência de HIV em gestantes e transmissão vertical segundo perfil socioeconômico, Vitória, ES. Rev Saúde Pública 2011; 45:644-51.

15. Domingues RMSM, Szwarcwald CL, Souza PRB, Leal MC. Prenatal testing and prevalence of HIV infection during pregnancy: data from the "Birth in Brazil" study, a national hospitalbased study. BMC Infect Dis 2015; 15:100.

16. Machado ACF, Sardinha JFJ, Ponte RL, Costa EP, Silva SS, Martinez-Espinosa FE. Prevalência de infecção por HIV, HTLV, VHB e de sífilis e clamídia em gestantes numa unidade de saúde terciária na Amazônia ocidental brasileira. Rev Bras Ginecol Obstet 2010; 32:176-83.

17. Szwarcwald CL, Barbosa Júnior A, Souza Júnior PRB, Lemos KRV, Frias PG, Luhm KR, et al. HIV testing during pregnancy: use of secondary data to estimate 2006 test coverage and prevalence in Brazil. Braz J Infect Dis 2008; 12:167-72.

18. Kupek E, Oliveira JF. Vertical transmission of HIV, syphilis and hepatitis B in the municipality with the highest incidence of AIDS in Brazil: a population-based study from 2002 to 2007. Rev Bras Epidemiol 2012; 15:478-87.

19. Miranda AE, Rosetti Filho E, Trindade CR, Gouvêa GM, Costa DM, Oliveira TG, et al. Prevalência de sífilis e HIV utilizando testes rápidos em parturientes atendidas nas maternidades públicas de Vitória, Estado do Espírito Santo. Rev Soc Bras Med Trop 2009; 42:38691.

20. Inagaki AD, Oliveira LAR, Oliveira MFB, Santos RCS, Araújo RM, Alves JAB, et al. Seroprevalence of antibodies for toxoplasmosis, rubella, cytomegalovirus, syphilis and HIV among pregnant women in Sergipe. Rev Soc Bras Med Trop 2009; 42:532-6.

21. Pereira G, Sabidó M, Caruso A, Oliveira S, Mesquita F, Benzaken A. HIV Prevalence among Pregnant Women in Brazil: A National Survey. Rev Bras Ginecol Obstet 2016; 38:3918.

22. Domingues RMSM, Saraceni V, Leal MDC. Mother to child transmission of HIV in Brazil: Data from the "Birth in Brazil study", a national hospital-based study. PLoS One 2018; 13:e0192985.

23. Miranda AE, Pereira GFM, Araujo MAL, Silveira MF, Tavares LDL, Silva LCF, et al. Avaliação da cascata de cuidado na prevenção da transmissão vertical do HIV no Brasil. Cad Saúde Pública 2016; 32:e00118215.
24. Anoubissi JD, Gabriel EL, Kengne NC, Fokam J, Tseuko DG, Messeh A, et al. Factors associated with risk of HIV-infection among pregnant women in Cameroon: Evidence from the 2016 national sentinel surveillance survey of HIV and syphilis. PLoS One 2019; 14:e0208963.

25. Rodrigues CS, Guimarães MDC, César CC. Missed opportunities for congenital syphilis and HIV perinatal transmission prevention. Rev Saúde Pública 2008; 42:851-8.

26. Kakehasi FM, Pinto JA, Romanelli RMC, Carneiro M, Cardoso CS, Tavares MCT, et al. Determinants and trends in perinatal human immunodeficiency virus type 1 (HIV-1) transmission in the metropolitan area of Belo Horizonte, Brazil: 1998-2005. Mem Inst Oswaldo Cruz 2008; 103:351-7.

27. Tornatore M, Gonçalves CV, Mendoza-Sassi RA, Silveira JM, D'ávila NE, Maas CG, et al. HIV-1 vertical transmission in Rio Grande, Southern Brazil. Int J STD AIDS 2010; 21:3515.

28. Gouveia PAC, Silva GAP, Albuquerque MFPM. Factors associated with mother-to-child transmission of the human immunodeficiency virus in Pernambuco, Brazil, 2000-2009. Trop Med Int Health 2013; 18:276-85.

29. Soeiro CMO, Miranda AE, Saraceni V, Lucena NO, Talhari S, Ferreira LCL. Mother-to-child transmission of HIV infection in Manaus, State of Amazonas, Brazil. Rev Soc Bras Med Trop 2011; 44:537-41.

30. Lana FCF, Lima AS. Avaliação da prevenção da transmissão vertical do HIV em Belo Horizonte, MG, Brasil. Rev Bras Enferm 2010; 63:58794.

31. Oliveira KWK, Oliveira SK, Barranco ABS, Hoffmann T, Duarte CS, Nazário RF, et al. Mother-to-child transmission of HIV in the Southern Region of Santa Catarina, from 2005 to 2015: analysis of risk factors to seroconversion in newborns. Rev Bras Saúde Mater Infant 2018; 18:461-9.

32. Araujo ESP, Friedman RK, Camacho LAB, Derrico M, Moreira RI, Calvet GA, et al. Cascade of access to interventions to prevent HIV mother to child transmission in the metropolitan area of Rio de Janeiro, Brazil. Braz J Infect Dis 2014; 18:252-60.

33. Zeng H, Chow EPF, Zhao Y, Wang Y, Tang M, Li L, et al. Prevention of mother-to-child HIV transmission cascade in China: a systematic review and meta-analysis. Sex Transm Infect 2016; 92:116-23.

34. Caffe S, Perez F, Kamb ML, Leon RGP, Alonso M, Midy R, et al. Cuba validated as the first country to eliminate mother-to-child transmission of human immunodeficiency virus and congenital syphilis: lessons learned from the implementation of the global validation methodology. Sex Transm Dis 2016; 43:733-6.

35. Lolekha R, Boonsuk S, Plipat T, Martin M, Tonputsa C, Punsuwan N, et al. Elimination of mother-to-child transmission of HIV - Thailand. Morb Mortal Wkly Rep 2016; 65;562-6. 
36. Tess B, Rodrigues L, Newell ML, Dunn D, Lago T. Breastfeeding, genetic, obstetric and other risk factors associated with mother-tochild transmission of HIV-1 in São Paulo State, Brazil. Aids 1998; 12:513-20.

37. Matida L, Santos NJ, Ramos A, Gianna M, Silva $M$, Domingues $C$, et al. Eliminating Vertical Transmission of HIV in São Paulo, Brazil: Progress and Challenges. J Acquir Immune Defic Syndr 2011; 57 Suppl 3:S164-70.

38. Ministério da Saúde. Agência Saúde. São Paulo elimina a transmissão do HIV de mãe para bebê. https://saude.gov.br/noticias/agencia-sau de/46005-sao-paulo-elimina-a-transmissaodo-hiv-de-mae-para-bebe (acessado em 30/ Mar/2020).
39. Barroso LMM, Galvão MTG, Cavalcante RM, Freitas JG. Cuidado materno aos filhos nascidos expostos ao HIV/AIDS. Rev Rene 2009; 10:155-64.

40. Freitas JG, Barroso LMM, Galvão MTG. Capacidade de mães para cuidar de crianças expostas ao HIV. Rev Latinoam Enferm 2013; 21:964-72. 


\section{Abstract}

Vertical HIV transmission is still an important global public health problem. This study aimed to verify vertical HIV transmission in Rio Branco, Acre, Brazil, and to assess the possibility of its elimination. A cross-sectional study was conducted of HIV in pregnant women and a longitudinal study on the incidence of vertical HIV transmission in pregnant women living in the municipality (county) of Rio Branco in 2007-2015. The cohorts of pregnant women consisted of women who had liveborn children, stillbirths, or abortions. The data were obtained from the Brazilian Information System for Notificable Diseases (SINAN), Brazilian Information System on Live Births (SINASC), Brazilian Mortality Information System (SIM), and Brazilian Hospital Information Systems (SIH). Databases. Probabilistic database linkage was performed with the OpenRecLink software. The authors calculated the HIV prevalence rate in pregnant women, the vertical transmission rate, and the principal associated factors. HIV prevalence in pregnant women showed an upward trend, and the mean prevalence was $0.18 \%$. Variables statistically associated with the occurrence of $H I V$ in pregnant women were maternal age $\geq 20$ years $(p=0.007)$, lower schooling $(p=0.054)$, and unmarried conjugal status/without partner ( $p=$ 0.001). Vertical HIV transmission was 6.9\%. Use of antiretroviral therapy (ART) during prenatal care, even among pregnant women that already knew they were HIV-positive, was less than $90 \%$. The elective cesarean rate was less than $60 \%$, and the use of ART during delivery and by the newborn in the first 24 hours showed variations, depending on the period in which the maternal diagnosis was made. Although the strategies for the elimination of vertical HIV transmission are well established, this study's results point to important flaws in the cascade of care for HIV-infected pregnant women in Rio Branco.

HIV; Pregnant Women; Prevalence; Vertical Infectious Disease Transmission

\section{Resumen}

La transmisión vertical del VIH todavía representa un importante problema de salud pública en el mundo. El objetivo de este estudio fue verificar la transmisión vertical del VIH en Río Branco-Acre y evaluar la posibilidad de su eliminación. Se realizó un estudio transversal de los casos de VIH en gestantes y longitudinal sobre la incidencia de la transmisión vertical del VIH en la base poblacional de gestantes residentes en el municipio de Río Branco, durante el período de 2007-2015. Las cohortes de gestantes estuvieron formadas por mujeres que tuvieron hijos nacidos vivos, muertos o abortos. Los datos se obtuvieron del Sistema Brasileño de Información de Enfermedades de Notificación (SINAN), Sistema de Información sobre Nacidos Vivos (SINASC), Sistema de Información sobre Mortalidad (SIM) y Sistema de Informaciones Hospitalarias $(\mathrm{SIH})$. Se realizó la relación entre las bases de datos, utilizando el software OpenRecLink. Se calcularon las prevalencias de VIH en gestantes, la tasa de transmisión vertical $y$ sus principales factores asociados. La prevalencia de VIH en gestantes presentó una tendencia de aumento y la prevalencia media fue de 0,18\%, las variables estadísticamente asociadas a la ocurrencia de VIH en gestantes fueron: edad materna $\geq$ 20 años $(p=0,007)$, menor escolaridad $(p=0,054)$ $y$ no contar con compañero $(p=0,001)$. La transmisión vertical fue de un 6,9\%. El uso de terapia antirretroviral viral (TARV) durante el período prenatal, incluso entre las gestantes que ya se sabian portadoras del virus, fue menor de un $90 \%$. La realización de cesáreas electivas quedó por debajo de un 60\% y el uso de TARV en el parto y por el recién nacido en las primeras 24 horas presentó variaciones, dependiendo del periodo en que el diagnóstico materno fue realizado. A pesar de que las estrategias de eliminación de la transmisión vertical del HIV estén bien establecidas, los resultados de este estudio todavía apuntan fallos importantes en la cascada de cuidados de las gestantes infectadas en Río Branco.

VIH; Mujeres Embarazadas; Prevalencia; Transmisión Vertical de Enfermedad Infecciosa

Recebido em 16/Abr/2020

Versão final reapresentada em 05/Ago/2020

Aprovado em 17/Ago/2020 